\title{
Avaliação de estratégias de gestão de ciência e tecnologia: um estudo de caso*
}

\author{
Assessment of science and technology management strategies: a case study
}

\author{
Júlio Cesar Rodrigues Pereira, Sueli Gonsalez Saes
}

Coordenação dos Institutos de Pesquisa da Secretaria de Saúde do Estado de São PauloSão Paulo, SP - Brasil

\begin{abstract}
A partir de implementaçāo de um modelo de gestão de ciência e tecnologia em institutos de pesquisa do Estado de Săo Paulo, Brasil, foi estudada a capacidade desse slstema para geraçāo de informaçōes gerenciais $\theta$ avaliado o incentivo à produçăo científica $\theta$ tecnológica, através do qual vem-se atribuindo bolsas a pesquisadores cujos projetos sắo selecionados através de uma revisāo por pares. Para avaliar a correçăo do processo de seleçăo de bolsistas, a produção científica média registrada em bases de dados de literatura nos últimos 5 anos, desses bolsistas, foi comparada por meio de análise de varlança a produçáo média de igual número de controles nāo-boisistas, pareados por instituto de origem. Foi estudada a capacidade do sistema de geréncia em produzir informaçőes por meio da apllcaçăo de análise por aglomeraçâ̌o. Os resultados mostraram que os bolsistas são pesquisadores que registram produção cientlflca significativamente superior aos não-bolsistas, permitindo inferir que a seieçāo de bolsistas esteja se processando adequadamente. Quanto ao potencial do sistema de gerência para produzir informaçōes, os resultados sugeriram que o processo de producçāo de pesquisa pode ser caracterizado com detalhes relevantes para a tomada de decisóes administrativas e de planejamento e que os institutos podem ser reunidos em grupos que comportam estratégias distintas de intervenção.
\end{abstract}

Apoio à pesquisa. Academias $e$ institutos, organização \& administração. Avaliaçāo de programas. Sistemas de informação administrativas.

\section{Introdução}

Nos últimos anos, a questão da ciência e tecnologia (C\&T) em saúde vem ganhando progressiva importância para a concepção e operação de sistemas de saúde. Após um período de ampla discussão sobre a matéria de ciência e tecnologia em saúde entre 1987 e 1989, especialistas de diferentes partes do mundo concluíram um documento ${ }^{3}$ de revisão do estado da arte, no campo, e já em 1990 a 43a Assembléia Mundial de Saúde ${ }^{19}$ aprovou resoluçăo $\left(\mathrm{n}^{\circ} 43.19\right.$, de 7.5.90) recomendando aos paísesmembros a adoção de medidas para a definição de prioridades para $C \& T$ em saúde, em nível nacional.

No Brasil, as atividades de C\&T em saúde são previstas na Constituição Federal: o artigo 218 atribui responsabilidade ao Estado para a promoção do desenvolvimento científico e tecnológico, e o inciso $\mathrm{V}$ do artigo 200 refere-se à matéria no âmbito específico do Sistema Único de Saúde. A Lei Orgânica da Saúde $\left(\mathrm{n}^{\circ} 8080 / 90\right)$ determina que o sistema de saúde cuide do "incremento, em sua área de atuação, do desenvolvimento cientifico e tecnologico" (inciso X, Artigo $6^{\circ}$ ) e indica como competências comuns da Uniāo, Estados e Municípios " $a$ realização de pesquisas e estudos na área da saú$d e "$ (inciso XIX, Artigo $15^{\circ}$ ). Entre os dias $24 \mathrm{e}$ 28.10.1994 realizou-se em Brasília a I Conferência Nacional de Ciência e Tecnologia em Saúde, quando foi dada a oportunidade para uma primeira e ampla discussāo da questão, tendo sido exaradas recomendaçōes de revisāo estrutural e funcional do Sistema de Saúde de forma a permitir-lhe cumprir suas tarefas no âmbito da ciência e tecnologia.

A administração da produção científica e tecnológica constitui-se em desafio gerencial porque esta produçāo se organiza por projetos e estes requerem uma estrutura administrativa matricial que conflita com o modelo vertical de gestāo existente para a produção de bens e serviços. A esse respeito, já em 1968, Cleland e $\mathrm{King}^{2}$ anotavam que na organizaçāo burocrática tradicional as atividades

\footnotetext{
* Projeto financiado pela Fundaçāo de Amparo à Pesquisa do Estado de Sdo Paulo (FAPESP), Processo ñ 94/3118-0. Separatas/Reprints: Júlio Cesar Rodrigues Pereira - Av. Dr. Amaldo, 351 - $1^{\circ}$ andar - 01246-901 - Săo Paulo, SP . Brasil Ediça subvencionada pelo FAPESP. Processo 95/2290-0.

Recebido em 25.11.1994. Reformulado em 7.6.1995. Aprovado em 27.6.1995.
} 
são conduzidas em movimentos de ascenção e descenso na hierarquia vertical, enquanto que o gerente de projetos está mais interessado nos fluxos de trabalho de direçôes horizontal e diagonal do que com qualquer fluxo de organização escalonada.

A despeito da tendência mundial de organização do trabalho de produção científica, tanto no ambiente acadêmico quanto no empresarial, no Brasil poucas são as iniciativas no campo e as existentes restringemse ao objeto geralmente denominado "pesquisa e desenvolvimento (P\&D)", sendo concebidas por empresas privadas num esforço de apoiar sua produção industrial ou comercial. Vasconcelos e Ohayon ${ }^{17}$, em 1989 , estudaram 27 institutos governamentais de pesquisa e 11 centros de $P \& D$ de empresas e em nenhum puderam identificar um sistema formal de administração de projetos. Kruglianskas ${ }^{10}$ (1987), analisou 51 empresas da região Sudeste do país, todas selecionadas pela sua importância na economia da regiāo, e encontrou que apenas $65 \%$ tínham procedimentos organizados de seleção de projetos e ainda que somente $35 \%$ tinham práticas padronizadas de acompanhamento e controle da execução de projetos. Como agravante, verificou que até $90 \%$ dos projetos eram descontinuados antes de alcançarem seus objetivos.

Enquanto uma abstração teórica que procura generalidades num ambiente altamente diversificado, a concepção de modelos gerenciais em C\&T (ou $P \& D)$ leva sempre uma marca de distância entre as idéias e o mundo real ${ }^{15}$. A Coordenação dos Institutos de Pesquisa da Secretaria de Saúde do Estado de Sāo Paulo (CIP), num esforço de oferecer resposta a este desafio, concebeu e implantou ao longo do ano de 1994, um modelo gerencial para projetos de pesquisa que compreendeu 3 módulos distintos:

Módulo 1: Cadastro de projetos de pesquisa. Este módulo estabelece um registro padronizado dos projetos de pesquisa que solicita a anotação do título da pesquisa, nome dos pesquisadores, resumo do projeto, fontes de financiamento do projeto e descrição das atividades do projeto divididas em diferentes fases de execução. Esta distinção de fases tem diferentes concepções entre autores desse campo ${ }^{8,16}$, mas constitui-se instrumento invariavelmente aplicado à gerência de projetos. As fases padronizadas são 6 :

Preliminar ou de concepção do projeto, que deve descrever atividades como definição de amostra ou material, definição de métodos, revisão do estado da arte, identificação de parcerias com indivíduos ou instituições para realização do estudo,entre outros. Pode envolver desde simples revisões da literatura até realização de workshops e seminários.

Preparatória, que deve descrever as atividades de testes-pilotos, calibragem de instrumentos, recrutamento e treinamento de pessoal de nível operacional, padronização de procedimentos, normas de registros de dados, e outras.

De campo, que deve descrever as atividades de coleta de dados e suas eventuais rotinas de supervisão, controle de qualidade e consistência de dados, rotinas de experimentação ou observação, e outras.

De análise, que deve descrever as atividades de processamento e análise dos dados coletados, testes de hipóteses, métodos e instrumentos para inferência de conclusões, e outras.

De redação, que deve descrever as atividades de produção de instrumentos de divulgação de resultados como produçâo de artigos científicos, resumos para congressos, elaboração de gráficos, tabelas, posters, slides, audiovisuais, manuais, livros, e outras.

De divulgação, que deve descrever as atividades previstas para divulgação de resultados, desde participação em congressos até elaboração de cursos, treinamentos, e outras.

Este módulo oferece aos institutos um software para registro padronizado das pesquisas e sugere a implantação de rotinas de seleção, orçamentação e acompanhamento da execução de projetos.

Módulo 2: Apoio à atividade de produção científica e tecnológica. Este módulo prevê a implantação de facilidades padronizadas de acesso e processamento de informações para pesquisa. No que se refere ao acesso, a CIP firmou acordo de cooperação com a BIREME (Centro Latino-Americano e do Caribe de Informaçôes em Ciências da Saúde) no sentido de integrar todas as bibliotecas dos Institutos de Pesquisa à rede informatizada da BIREME. Os recursos financeiros necessários foram repassados à BIREME em junho/94 e a implantação do sistema foi concluída em dezembro/94. Quanto ao processamento de dados, está programada a implantação de uma rede local para cada Instituto, com conexão à rede internacional INTERNET.

Módulo 3: Incentivo à produçāo científica e tecnológica. Constitui-se num programa de atribuição de bolsas a pesquisadores, cujas pesquisas, avaliadas através derevisão por pares (peer review), pela Comissão Científica da CIP, sejam identificadas como de mérito em qualidade técnico-científica e em identidade com as necessidades do Sistema Único de Saúde. As bolsas são uma forma de reconhecimento pecuniário à qualidade da produção científica e tecnológica e, têm vigência pelo prazo de execução do projeto de pesquisa e seu valor varia segundo a qualificação acadêmica do beneficiário. O programa é gerenciado administrativamente pela FUNDAP (Fundação de Apoio à Administração Pública do Estado de São Paulo), através de contrato estabelecido para este fim, a 
exemplo de outros programas de bolsas da Secretaria de Saúde que lhe são confiados.

$O$ presente estudo pretende avaliar a iniciativa de gestão de ciência e tecnologia experimentada na Secretaria de Saúde do Estado de São Paulo. Seu objetivo é explorar a capacidade potencial dos dados gerados por esta iniciativa na produção de informações gerenciais e avaliar a propriedade com que o programa de bolsas para pesquisadores foi realizado.

\section{Metodologia}

A produção científica e tecnologica de pesquisadores e instituições de pesquisa não conhece $p a-$ drōes-de-ouro objetivos que permitam comparações definitivas. Fahrni e Spatig ${ }^{5}$, revendo métodos de avaliação deste tipo de produção, propuseram 12 diferentes modelos para trabalhar variáveis quantitativas e qualitativas. Seus protótipos são montados levando em conta o grau de quantificação da informação sobre os projetos, a interdependência de recursos entre projetos, a caracterização dos objetivos como únicos ou múltiplos e, ainda, criando scores que com diferentes pesos levam em conta a viabilidade tecnológica, a atratividade técnica e o potencial econômico dos projetos.

Miller ${ }^{13}$ realizou estudo de validação da análise bibliométrica (registro de publicaçōes em bases de dados de literatura) e demonstrou que a medida do número de publicações é um indicador confiável, com bom nível de concordância com outras alternativas, para a avaliação da produção científica e tecnológica de instituições de pesquisa. LuukkonenGronow $^{12}$, em estudo de métodos de avaliação de sistemas de gestão em pesquisa e desenvolvimento, destacou que se deve reconhecer além de variáveis de resultados, também as de potencial e de processo que caracterizem a execução dos projetos.

Com base nesses argumentos, no presente trabalho foi avaliado o programa de bolsas da CIP, partindo da premissa de que o volume de publicações é um indicador, ainda que indireto, da qualidade da produção científica e tecnológica, e que pode ser levantado através de consulta a bases de dados da literatura. Tomando em conta a pertinência de cotejar-se estas variáveis de resultado com caraterísticas de potencial e processo de cada instituto. propôs-se ainda processar os dados coletados com vistas a oferecer tanto uma descrição do perfil de cada instituto quanto uma investigação de suas possíveis tendências de agrupamento.

Para avaliar se de fato o programa de bolsa havia premiado os pesquisadores de maior qualificação técnico-científica, os 85 bolsistas existentes no programa tiveram sua produção científica, em termos de número de publicações registradas nos últimos 5 anos, (1990-1994) comparada à produção científica de 85 pesquisadores-controles, pareados por instituto de origem. A seleção de controles foi feita de forma aleatória através de programa de computador (preparado em linguagem Clipper) que, pesquisando as bases de dados (preparada em DBASE-III/+) contendo os nomes de todos os profissionais de nível universitário sorteou controles em número idêntico ao de bolsistas para cada instituto.

A produção científica de bolsistas e controles foi levantada, sob aconselhamento e orientação de técnicos da Biblioteca da Faculdade de Saúde Pública da USP. A pesquisa bibliográfíca foi feita em 4 bases de dados no formato CD-Rom (compact disk - ready on memmory), para o período de 1990-1994. Essas bases foram: MEDLINE*, e LILACS** que cobrem principalmente o campo das ciências da saúde; Life Sciences Collection ${ }^{* * *}$, que cobre principalmente a produção em ciências biológicas básicas; e Food Science Tecnology Abstracts****, que cobre principalmente publicaçōes do campo da toxicologia de alimentos. Os autores foram identificados através de todas as combinações previstas em cada base de dados, para nome e sobrenome; os homônimos foram excluídos pela verificação de consistência dos nomes com endereço institucional, campo de investigação e confíguração de equipe de pesquisa. Estas duas últimas observaçôes se fizeram necessárias porque identificaram-se registros em que o autor, por ser co-autor ou por definições de equipe de investigação, constava na publicação com endereço diferente de sua instituição de origem. Sempre que um registro mostrou inconsistência simultaneamente de endereço, de campo de investigação e de composição de equipe, considerou-se a ocorrência de homônimo e o registro foi desconsiderado para o autor pesquisado.

A comparação da produção de bolsistas com a de controles foi feita através de análise de variâncial (oneway ANOVA) contrapondo-se a média registrada para cada grupo através de intervalos de confiança de $95 \%$ e calculando-se a probabilidade de ocorrência de diferença devida ao acaso. Estabeleceu-se de antemão o nível de significância de $\mathrm{p}=.05$ para rejeitar-se a hipótese nula de igualdade entre bolsistas e controles.

A caracterização das condições de potencial e de processo dos institutos foi feita pelo reconhecimento do potencial de recursos humanos para pesquisa, do número de pesquisas registrado pelo Sistema de

* MEDLINE - Medical Literature on Line, editada pela National Librar: of Medicine.

** LILACS-Literatura Latino-Americano em Ciências de Saúde, editada pela BIREME.

*** LIFE SCIENCES COLLECTION, editada pela Cambridge Scientific Abstracts.

**** FOOD SCIENCE TECNOLOGY ABSTRACTS, editada pela International Food Information Services. 
Gerência da CIP, pelo tempo médio de execução de projetos (time horizon) e pelo tempo médio de cada fase de execução do projeto, também recuperados do Sistema de Gerência da CIP. Além da descrição destas variáveis para contraposição à produção média dos institutos, registradas pela amostra de bolsistas e controles, exploram-se possíveis associaçðes entre as variáveis calculando-se o $r$ de Pearson e sua significância estatística ${ }^{18}$. Procedeu-se ainda a uma análise de aglomeraçăo (Cluster analysis ${ }^{6}$ ) dos institutos examinando-se suas distâncias em relação tanto às variáveis de produção (número médio de publicações nos últimos 5 anos, per capita) quanto às de potencial e processo (número de profissionais universitários, número de projetos registrados $\mathrm{e}$ tempo médio de execução de projetos). Foi utilizado o método de aproximação centróide (average linkage) para aglomeração e utilizam-se distâncias Euclidianas de resíduos padronizados da média das variáveis processadas.

Todo o processamento estatístico foi realizado com auxflio do pacote SPSS/PC+, versão 4.0 (Statistical Package for Social Sciences).

\section{Resultados}

A Tabela 1 mostra o número de pesquisas e o tempo médio previsto para sua execuçāo por instituto de pesquisa. A Figura 1 descreve as tendências de distribuição deste tempo de execução para cada fase de atividades do projeto. As pesquisas parecem ser em geral de longa duração e embora haja uma predominância do tempo dedicado à fase menos flexível da execução de um projeto, a fase de campo onde se realizam experimentos ou se observam eventos, parece haver margem para uma diminuiçåo do tempo total de pesquisa se as outras fases do processo de pesquisa puderem ser aceleradas. Isto
Tabela 1 - Número de pesquisas e tempo médio de execução nos Institutos de Pesquisa da Secretária de Saúde de São Paulo.

\begin{tabular}{ccc}
\hline $\begin{array}{c}\text { Instituto de } \\
\text { Pesquisa }\end{array}$ & $\begin{array}{c}\text { Número de Pesquisas } \\
\text { Cadastradas em 1994 }\end{array}$ & $\begin{array}{c}\text { Tempo Médio } \\
\text { de Execuçáo }\end{array}$ \\
\hline Adolfo Lutz & 240 & 37 meses \\
Butantan & 109 & 55 meses \\
Emílio Ribas & 53 & 35 meses \\
Inst. de Saúde & 43 & 32 meses \\
Dante Pazzanese & 40 & 26 meses \\
Lauro Souza Lima & 18 & 30 meses \\
Pasteur & 9 & 18 meses \\
\hline
\end{tabular}

Fonte: Sistema de Geréncia de Pesquisas da CIP

dependeria menos de qualquer reorientação político-institucional e mais da consolidação de uma infra-estrutura de apoio à atividade de pesquisa.

Uma eventual reduçāo no tempo médio de execução de pesquisa talvez permitisse o engajamento dos institutos num maior número de pesquisas, embora os dados nảo sugiram que o tempo de execução seja fator restritivo ao número de projetos: não há correlaçāo entre as variáveis $(r=0,52, p=0,24)$.

Tampouco o número de pesquisadores em potencial em cada instituto, apresentado na Tabela 2 , tende a explicar o número de pesquisas por instituto ( $r=0,64, p=0,12$ ). De fato, os compromissos com os serviços que os institutos prestam diretamente ao sistema de saúde freqüentemente privam os institutos de pesquisa de um razoável contingente para a produção científica e tecnológica. Há que se registrar, contudo, que à exceçāo dos Institutos de Saúde, Lauro de Souza Lima e Pasteur, uma reserva não desprezível de recursos humanos pode ter seu trabalho organizado e fomentado para uma maior produçăo científica e tecnológica.

Processados os dados relativos à produção científica dos últimos 5 anos para bolsistas e controles os

№ MÉDIO DE DIAS

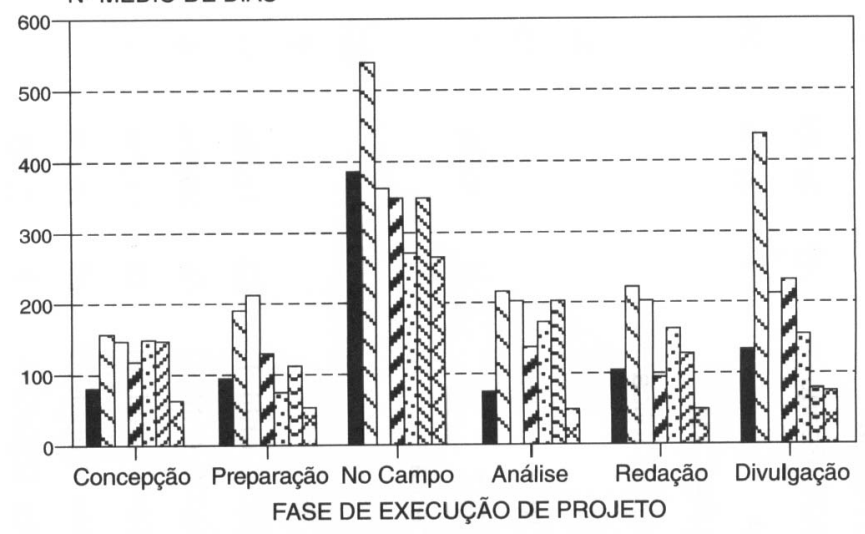

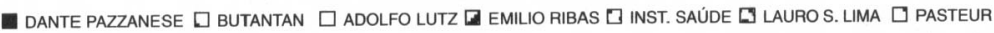

Figura 1 - Tempo médio das diferentes fases de execução de projetos de pesquisa nos institutos da Secretaria de Saúde do Estado de Såo Paulo. 


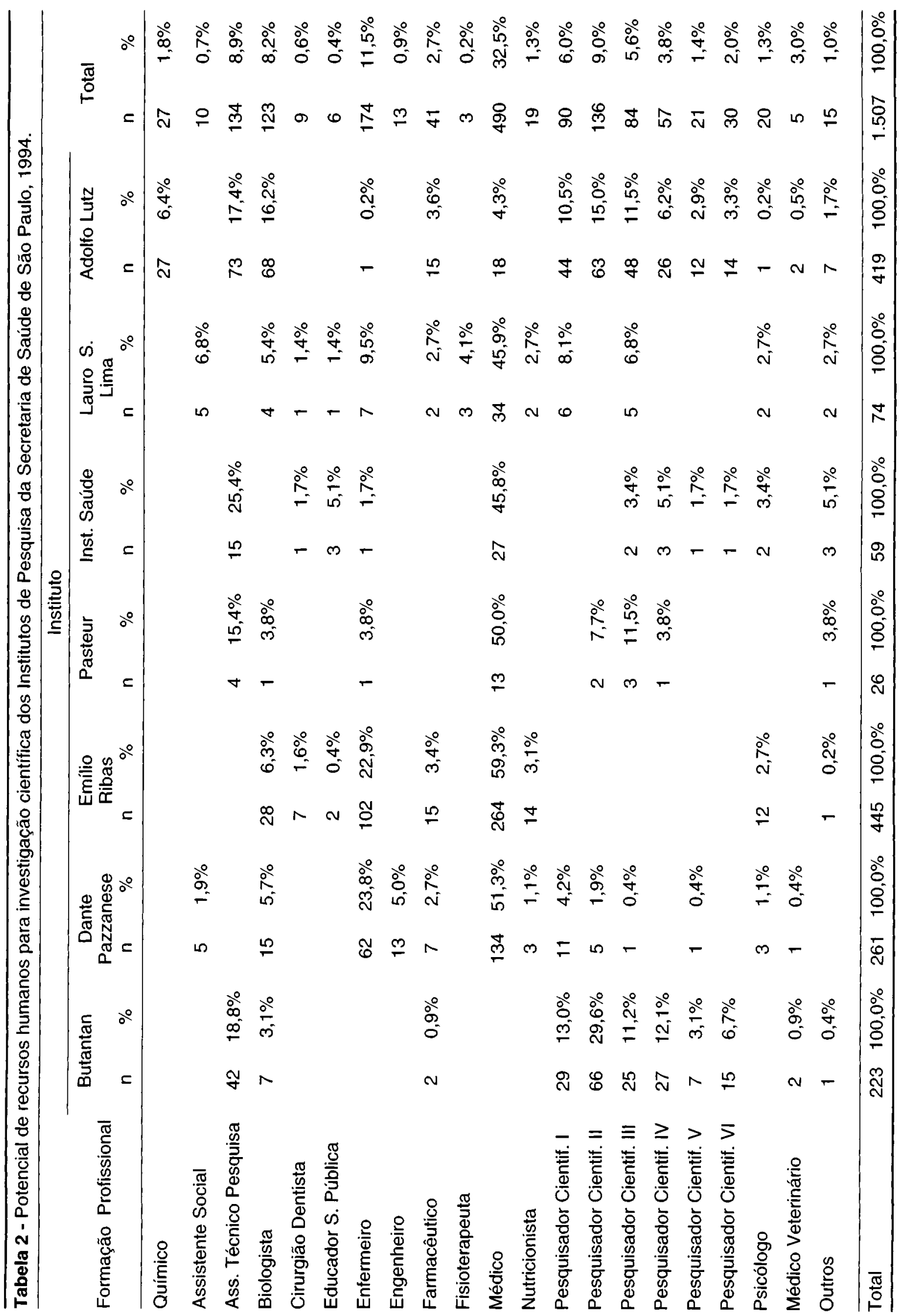


resultados obtidos estão apresentados na Figura 2.

À exceção da base de dados FOOD SCIENCE, onde embora bolsistas superem controles há superposição dos intervalos de confiança, encontrou-se marcada distinção entre bolsistas e seus controles. Na Tabela 3, a comparação é dissociada por instituto de pesquisa e, à exceção da base de dados LIFE SCIENCES para o Instituto de Saúde, os bolsistas superam invariavelmente seus controles, embora em nível de cada instituto por vezes o número de observações seja muito pequeno para garantir significância estatística a estas diferenças. Na comparaçāo total de casos e controles, a informação já apresentada na Figura 2 traduzida em números. A Tabela 3 presta-se também à comparação entre os institutos, ressalvada a representatividade da amostra que foi desenhada apenas para a comparação da produçāo científica de bolsistas e controles.

Assumido este limite da amostra e dentro do escopo proposto para o presente estudo, de estender a utilizaçāo dos dados coletados para uma exploração das alternativas de análise propiciadas por um sistema de gestão em ciência e tecnologia, procedeu-se à análise de aglomeração dos institutos com utilização das medidas de produçāo das quatro bases de dados da literatura, o número de pesquisadores em potencial por instituto, o número de pesquisas registradas por instituto e seu tempo médio de execução. Os resultados do processamento são apresentados na Tabela 4.

A matriz dos coeficientes de dissimiliaridade mostra que a distância de seus pares, no Instituto Adolfo Lutze Instituto Butantan é expressivamente maior do que aqueles entre si, e tanto os coeficientes exibidos no esquema de aglomeração quanto a sequiência de ligação dos institutos, exibida pelo dendograma, sugerem que o melhor esquema de aglomeraçāo seria provavelmente aquele que distinguisse 3 grupos. Na Tabela 5, comparam-se as médias das variáveis utilizadas para a aglomeração dos institutos em 3 grupos.

$O$ agrupamento sugere que o Instituto Adolfo Lutz e o Instituto Butantan se distingam dos outros pela maior produtividade e que sejam também distintos entre si, principalmente pelas diferenças de campos de investigaçăo. De fato, entre as variáveis de potencial e processo parece haver uma compensaçāo de forma que aquele que registra maior número de pesquisas tem também maior número de pesquisadores e menor tempo de execuçāo. Menos que diferenças produtivas, essas caraterísticas parecem delinear diferentes espectros de ciência e tecnologia, como de fato confirma o exame do número de publicações por base de dados: enquanto o Insituto Butantan supera o Instituo Adolfo Lutz.nas ciências da saúde (MEDLINE) é, no entanto, por ele superado provavelmente em algum campo das ciências básicas (LIFE SCIENCES), talvez na saúde coletiva (que tem contribuição mais expressiva no LILACS) e no campo da toxicologia de alimentos (FOOD SCIENCES), que é específico do Adolfo Lutz.

Os outros institutos agrupam-se pela menor produtividade. Embora o Instituto Dante Pazzanese se destaque em produtividade, e em certa medida se destaque tambem o Pasteur (MEDLINE), suas produçōes mais elevadas contrapostas a seus igualmente maiores potenciais acabam por colocá-los em igualdade de condições com seus outros pares.

\section{BASES DE DADOS}

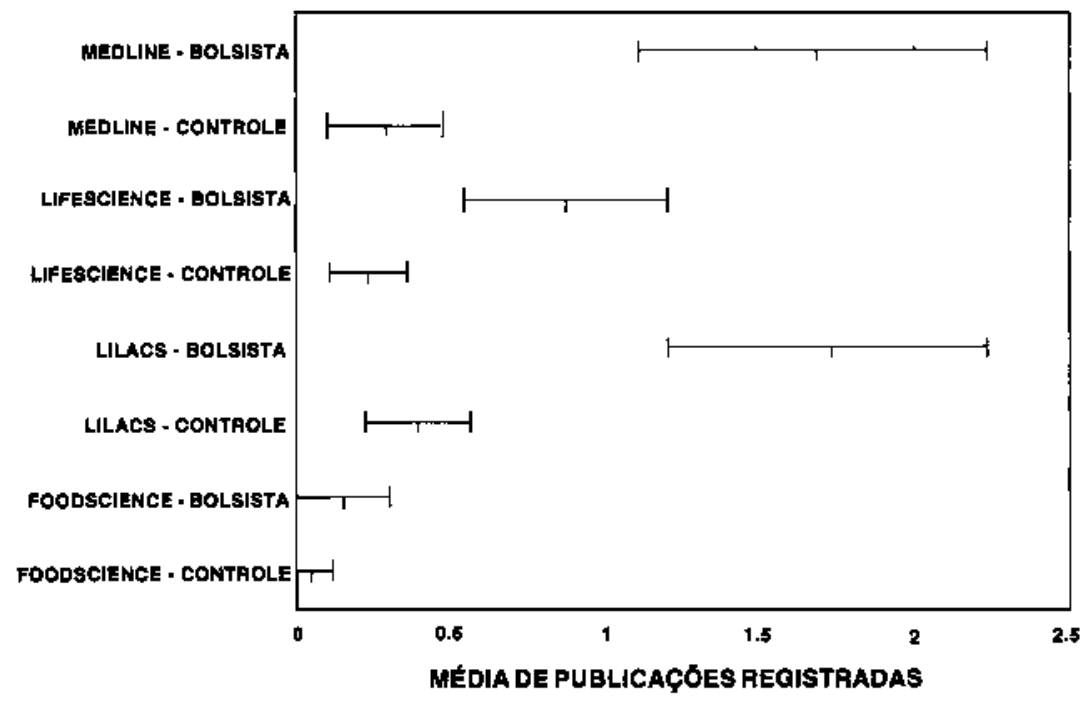

I MAXIMO I MÍNINO - MÉOIA

Flgura 2 - Número médio de publicaçōes per capita para bolsistas e controles nos últimos períodos de 1990/ 1994 e intervalo de confiança de $95 \%$. 
Tabela 3 - Número médio de publicações registradas em diferentes bases de dados no perlodo de 1990-94, para Bolsistas e Controles \& Probabilidade de Diferença devida ao acaso.

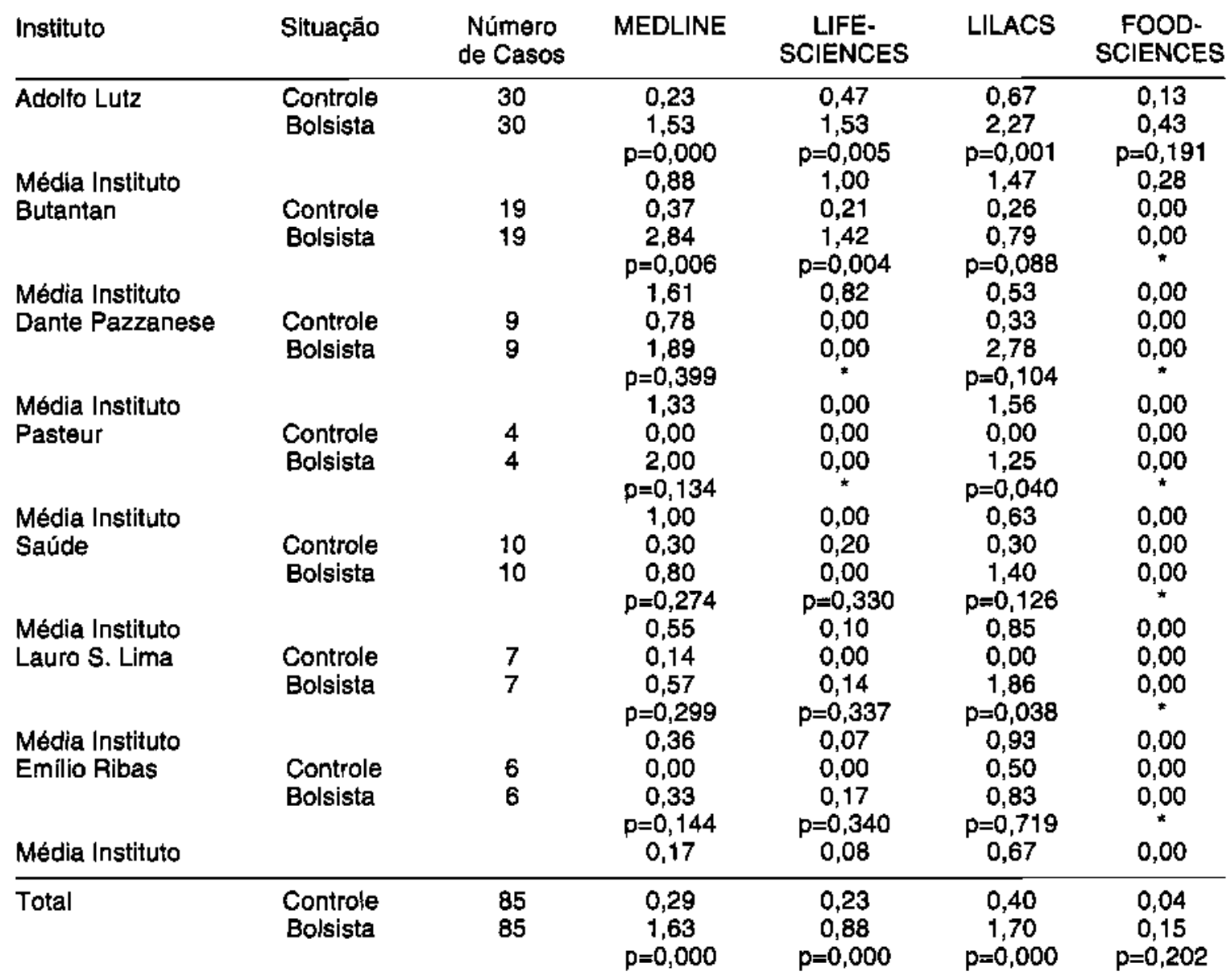

* = Cálculo de probabilidade năo se aplica ou não pode ser realizado

Enquanto os grupos 1 e 2, ou o Instituto Adolfo Lutz e o Instituto Butantan, talvez possam se beneficiar principalmente de intervençőes em nível dos processos, eg. reduçāo do tempo médio de execução de projetos, os institutos do grupo 3 , cujo tempo médio de execuçāo de projetos é de 9 a 27 meses inferior aos outros dois grupos, parecem requerer alguma intervenção que amplie seus potenciais. Compare-se na Tabela 2 que, enquanto no Butantan e no Adolfo Lutz os pesquisadores científicos com dedicação exclusiva à pesquisa somam cerca de 75 e 50\%, respectivamente, nos outros institutos esta proporção sequer atinge os $15 \%$. A análise de variância da produçăo científica por categoria profissional revelou desigualdades estatisticamente significantes $(F<.01)$ em todas as bases de dados $\mathrm{e}$ a polarização foi sempre devida à maior produção dos pesquisadores científicos IV, V e VI que invariavelmente se destacaram das outras categorias, embora nem sempre fossem diferentes entre si.

\section{Comentários e Conclusōes}

A análise dos dados sugere que o programa de bolsas do sistema de gerência da CIP selecionou, de fato, com acerto os melhores pesquisadores dos institutos para a atribuição do benefício. $O$ fato de não se ter observado diferença estatisticamente significante entre bolsistas e controles na base de dados FOOD SCIENCES, nāo chega a prejudicar esta conclusão, tanto porque se trata de um campo específico onde se registra um pequeno número de dados para análise quanto pelo fato de que a hipotese nula de igualdade entre bolsistas e controles só não é descartada pelo rigor assumido para o nível de significância de $5 \%$. A Tabela 3 registra que a probabilidade de bolsistas e controles terem produção idêntica é de $20 \%$ o que significa, em contrapartida, que a probabilidade de acerto do programa de bolsas é, ainda neste caso, de $80 \%$.

Kingsbury ${ }^{9}$, já em 1967, afirmava que estratégias 
Tabela 4 - Resultado da análise de Cluster para agrupamento dos Institutos de Pesquisa da Secretaria de Saúde do Estado São Paulo.

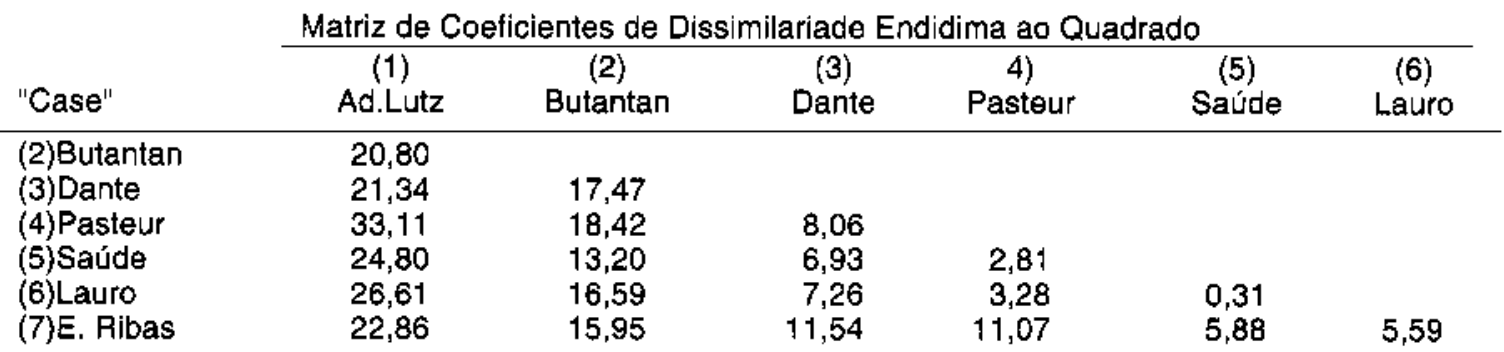

Esquema de aglomeração usando conexão média (entre grupos)

(Entre grupos)

Estágio
1
2
3
4
5
6

Aglomerado

\begin{tabular}{rrr}
\hline Cluster 1 & Cluster 2 & Coeficiente \\
\hline 5 & 6 & 0.313443 \\
4 & 5 & 3.050740 \\
3 & 4 & 7.424416 \\
3 & 7 & 8.527666 \\
2 & 3 & 16.332260 \\
1 & 2 & 24.923311
\end{tabular}

Dendrograma usando conexão média (entre grupos)

Distância reescalonada da combinaçāo de aglomerados

"C ASE

Label Seq."

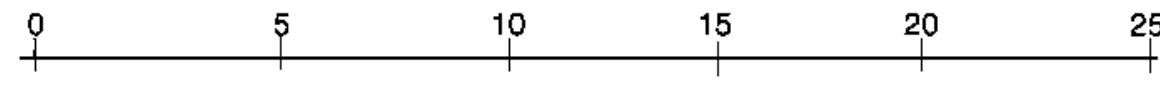

Saúde

Lauro

Pasteur

Dante

E. Ribas

Butantan

Ad. Lutz

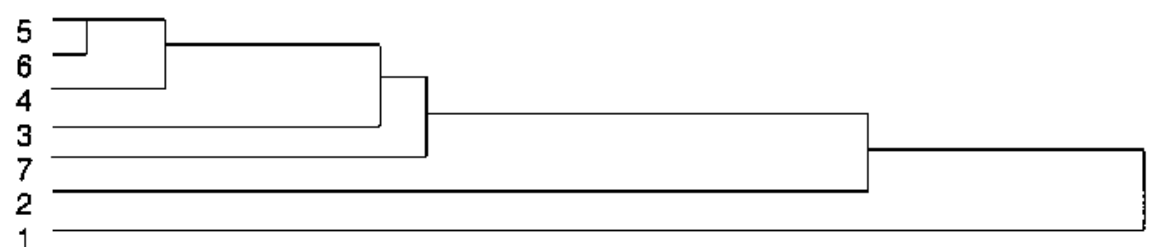

Tabela 5 - Caracterização dos grupos de Institutos de Pesquisa de acordo com as médias das variáveis utilizadas, período 1990-94

\begin{tabular}{llllllccc} 
Grupo Instituto & $\begin{array}{c}\text { MED } \\
\text { LINE }\end{array}$ & $\begin{array}{c}\text { LIFE } \\
\text { SCIENCES }\end{array}$ & & LILACS & $\begin{array}{c}\text { FOOD } \\
\text { SCIENCES }\end{array}$ & $\begin{array}{c}\text { Número } \\
\text { Pesquisadores }\end{array}$ & $\begin{array}{c}\text { Número } \\
\text { Pesquisas }\end{array}$ & $\begin{array}{c}\text { Tempo } \\
\text { Médio } \\
\text { Execução } \\
\text { (meses) }\end{array}$ \\
\hline 1 & Adolfo Lutz & 0,88 & 1,00 & 1,47 & 0,28 & 419,00 & 240,00 & 37,00 \\
2 & Butantan & 1,61 & 0,82 & 0,53 & 0,00 & 223,00 & 109,00 & 55,00 \\
3 & Dante Pazzanese & 1,33 & 0,00 & 1,56 & 0,00 & 261,00 & 40,00 & 26,00 \\
& Pasteur & 1,00 & 0,00 & 0,63 & 0,00 & 26,00 & 9,00 & 18,00 \\
& Saúde & 0,55 & 0,10 & 0,85 & 0,00 & 59,00 & 43,00 & 32,00 \\
& Lauro S. Lima & 0,36 & 0,07 & 0,93 & 0,00 & 74,00 & 18,00 & 30,00 \\
& Emílio Ribas & 0,17 & 0,08 & 0,67 & 0,00 & 445,00 & 53,00 & 35,00 \\
& Média do Grupo & 0,68 & 0,05 & 0,93 & 0,00 & 173,00 & 32,60 & 28,20 \\
\hline Média dos Institutos & 0,84 & 0,30 & 0,95 & 0,04 & 215,29 & 73,14 & 33,29
\end{tabular}

de recompensas e incentivos podiam incrementar a produção de estudos e pesquisas. Henrichs, em um estudo que investigou as expectativas de pesquisadores para seu ambiente de trabalho, definiu uma lista de 10 itens a serem observados na promoção do trabalho acadêmico/científico e uma boa remuneração estava entre eles. Ellis e HonigHaftel $^{4}$, em um estudo que envolveu entrevistas com gerentes de 33 grandes centros de pesquisa e 111 pequenos, demonstraram que prêmios de altas somas 
em dinheiro podem resultar em um aumento de registro de patentes da ordem de $48 \%$. Ao programa de bolsas da CIP restará ainda a necessidade de um acompanhamento prospectivo que afira o impacto deste tipo de incentivo na produção científica dos beneficiários.

De fato, as conclusões do presente estudo limitam-se a validar o mérito da distribuiçåo de bolsas sem alcançar a avaliação do mérito das próprias bolsas como estratégia de incentivo à produção científica. Num desenho de comparaçăo de casos e controles, para além do recebimento de bolsas, outros "fatores de exposiçāo" precisariam ser cotejados como potenciais variáveis de confusão, destacando-se ai antecedentes pessoais e profissionais que não foram considerados por indisponibilidade de informação. Nesta situação, apenas um acompanhamento prospectivo poderá, no futuro, através da comparaçăo de situaçōes ex-ante e ex-post, confirmar a associaçăo aqui encontrada bem como definir-lhe a direção de causa e efeito. Embora para o programa de bolsas e particularmente para a Comissão Científica da CIP, encarregada da seleçāo de bolsistas, a conclusão da presente avaliaçăo seja inegavelmente relevante, pois que valida seu trabalho, por outro lado, ao sistema de gerência da CIP, como um todo, será muito importante avaliações futuras que verifiquem a efetividade do programa enquanto estratégia de incentivo.

O processamento exploratório dos dados recolhidos para o objetivo específico do estudo permitiu, senão uma identificação definitiva das tendências da produção científica e tecnológica dos institutos, pelo menos uma demonstração da potencialidade de geraçāo de informaçōes que o sistema de gerência de pesquisa pode propiciar. $O$ reconhecimento de distintos perfis de espectro de ciência e tecnologia entre os institutos e suas possibilidades de agrupamento são informaçôes de todo relevantes para os processos de tomada de decisão. As evidências de que o tempo de execução de projetos pode ser abreviado através de iniciativas de apoio às atividades de planejamento, processamento e finalização da pesquisa sugerem que as açōes previstas pelo módulo 2 do sistema de gerência sāo oportunas e adequadas. Possa ser o módulo 1, de cadastro de pesquisas, adequadamente utilizado na seleção e acompanhamento de projetos e como conseqüência natural poder-se-ia esperar um incremento da produção científica dos institutos.

$O$ fato dos institutos com maior contingente de pesquisadores científicos com dedicação exclusiva à pesquisa serem também os de maior produtividade acadêmica, aliado à informação de que estas categorias têm produçāo significativamente maior que as outras, sugere claramente que ou os institutos restantes devam receber uma maior alocação destes profissionais ou que seu regime de trabalho deva ser emulado para outras categorias. Esta última hipotese é particularmente importante para o caso dos médicos, categoria que em 5 dos 7 institutos representa $45 \%$ ou mais dos quadros para pesquisa (Tabela 2), cuja fixação em tempo integral nos institutos poderia vir a ter importante impacto na sua produtividade. A concessão de bolsas deve ser um passo no sentido da fixação de profissionais na medida em que o benefício os desonerem de outros compromissos, mas certamente não será solução suficiente até porque seus valores foram concebidos apenas para premiar a qualidade e nāo para efetivamente complementar salários.A gestāo de ciência e tecnologia, para além das dificuldades inerentes à matéria, padece ainda da resistência ou da indiferença da comunidade acadêmica. Vasconcelos e Ohayon $^{17}$, em 1989, tendo estudado empresas privadas e institutos de pesquisa, encontraram que os diretores destas instituições não consideravam prioritária a implementação de sistemas de avaliaçăo em $\mathrm{P} \& \mathrm{D}$ e concluíram que era a ausência de sistemas formais que concedia liberdade de arbítrio a cada nível gerencial com prejuízo em última instância da própria parte interessada, mas resistente. Uma publicação recente patrocinada pelo Ministério da Ciência e Tecnologia ${ }^{14}$, numa extensa revisão das tendências de $C \& T$ no Brasil, identifica esta resistência da comunidade acadêmica em estabelecer diálogo com as instâncias administrativas $\mathrm{e}$ aponta a necessidade de superar esta barreira com vistas a um desejado desenvolvimento no campo da ciência e tecnologia.

Lilien ${ }^{11}$ observou que administradores demandam simplicidade, certeza, imediatismo e concretismo enquanto que pesquisadores oferecem complexidade, probabilidade, futurismo e abstração. Para que a ciência e tecnologia venham efetivamente constituir-se num campo específico do sistema de saúde, administradores e pesquisadores deverão aprender a colaborar.

\section{Agradecimento}

Ao staff da Biblioteca da Faculdade de Saúde Pública pela gentileza e facilidades oferecidas para recuperaçāo dos dados bibliográficos.

\section{Referências Bibliográficas}

1. ARMITAGE, P. \& BERRY, G. Statistical methods in medical research. London, Blackwell Sci. Publ,, 1987.

2. CLELAND, D. I, \& KING, W. R. System analysis and project management. New York, McGraw-Hill, 1968.

3. COMMISSION ON HEALTH RESEARCH FOR DEVELOPMENT. Health research: essential link to equity in development. New York, Oxford University Press, 1990.

4. ELLIS, L. W. \& HONIG-HAFTEL, S. Reward strategies for R \& D, Res. Technol. Manag., 22: 16-20, 1992.

5. FAHRNI, P. \& SPATIG, M. An application-oriented guide to 
$\mathrm{R} \& \mathrm{D}$ project selection and evaluation methods. $R \& D$ Manag., 20: 155-71, 1990.

6. HAIR Jr, J. F.; ANDERSON, R. E.; TATHAM, R. L. Multivariate data analysis. New York, MacMillan Publ. Co., 1987.

7. HENRICHS, J.R. Creativity in industrial research. In: Blood, J.W. ed. The management of scientific talent. New York, American Management Association, 1976. p. 155-78.

8. KING, W. R \& CLELAND, D. I. Life cicle management. Apud Cleland, D. I, \& King W, R. System analysis and project management. New York, McGraw-Hill, 1968.

9. KINGSBURY, S. Behind the organizational chart. In: Hainer, R. M.; Kingsbury, S.; Gleicher, D. B. Uncertainty in research management and new product development. New York, Reinhold Publ. 1967. p. 26-55.

10. KRUGLIANSKAS, I. Seleçăo, planejamento e controle de projetos de P\&D: um estudo exploratorio em empresas brasileiras. Săo Paulo, 1987 [Tese de Livre Docência Faculdade de Economia e Administração da LSP].

11. LILIEN, G. L. MS/OR: A mid-life crisis. Inferfaces, 17(2): $35-8,1987$.

12. LUUKKONEN-GRONOW, T., Scientific research evaluation; a review of methods and various contexts of their application. $R \& D$ Manag. 17:
207-21, 1987.

13. MILLER, R. The influence of primary task on $R$ \& $D$ laboratory evaluation: a comparative bibliometric analysis. $R \& D$ Manag., 22: 3-20, 1992.

14. SCHWARTZMAN, S. coord. Ciência e tecnologia no Brasil: uma nova política para um mundo global. Sâo Paulo, Fundação Getúlio Vargas, 1993.

15. SOUDER, W. E. A sy'stem for using $R \& D$ project evaluation models in organizations. Pittisburgh, Techonlogy Management Studies Group, University of Pittisburgh, Dept. of Industrial Engineering, 1978.

16. TUMAN Jr, J. Development and implementation of effective project management information and control systems. In: Cleland, D. I. \& King, W. R. Project management handbook. New York, Van Nostrand Reinhold Co., 1983. p. 495-529.

17. VASCONCELLOS, E. \& OHAYON, P. Como avaliar projetos de pesquisa e desenvolvimento tecnológico? Rev. Adm., 24(3): 31-8, 1989.

18. WOODWARD, M. \& FRANCIS, L. M. A. Statistics for health management and research. London. Edward Arnold, 1988.

19. WORLD HEALTH ORGANIZATION. 43. World Health Assembly. Genevs, 1990.

\begin{abstract}
The Health Department of the State of S. Paulo has implemented a management strategy on research and development (R\&D) for its research institutes which comprises three different items: 1) project registration, 2) support and 3) promotion of R\&D activities. The present study explores the ability of this R\&D management system to provide information and evaluates the performance of the third item which has been responsible for the assignment of research grants to professionals whose projects are selected through a peer review. The assessment of the grant's distribution was conducted by comparing the mean scientific production recorded in literature data bases for grant holders and equal an number of controls matched by institute of origin. The ability of the system to produce intormation was studied by the application of Ciuster Analysis. The results show that grant holders have a scientific production significantiy higher than their controls. Conceming information production, it is shown that proper raw data processing may render suggestions for decision making and that the institutes may be grouped in clusters of those that adopt similar management strategies.
\end{abstract}

Research support. Academies and Institutos, organization and administration. Program evaluation. Management Information Systems. 